\title{
The role of molecular chaperones in clathrin mediated vesicular trafficking
}

\author{
Rui Sousa and Eileen M. Lafer* \\ Department of Biochemistry and Center for Biomedical Neuroscience, University of Texas Health Science Center at \\ San Antonio, San Antonio, TX, USA
}

\section{OPEN ACCESS}

Edited by:

Matthias Peter Mayer,

Ruprecht-Karls-Universität

Heidelberg, Germany

Reviewed by:

Rafael Fernández-Chacón,

The Neuroscience Institute at Seville,

Spain

Dana Reichmann,

The Hebrew University of Jerusalem,

Israel

${ }^{*}$ Correspondence:

Eileen M. Lafer,

Department of Biochemistry,

University of Texas Health Science

Center at San Antonio, 7703 Floyd

Curl Drive, Room 415B, San Antonio,

TX 78229, USA

lafer@uthscsa.edu

Specialty section:

This article was submitted to

Protein Folding, Misfolding and

Degradation

a section of the journa

Frontiers in Molecular Biosciences

Received: 09 April 2015

Accepted: 05 May 2015

Published: 19 May 2015

Citation:

Sousa $R$ and Lafer EM (2015) The role

of molecular chaperones in clathrin mediated vesicular trafficking.

Front. Mol. Biosci. 2:26.

doi: 10.3389/fmolb.2015.00026
The discovery that the $70 \mathrm{kD}$ "uncoating ATPase," which removes clathrin coats from vesicles after endocytosis, is the constitutively expressed Hsc70 chaperone was a surprise. Subsequent work, however, revealed that uncoating is an archetypal Hsp70 reaction: the cochaperone auxilin, which contains a clathrin binding domain and an Hsc70 binding $\mathrm{J}$ domain, recruits Hsc70*ATP to the coat and, concomitant with ATP hydrolysis, transfers it to a hydrophobic Hsc70-binding element found on a flexible tail at the C-terminus of the clathrin heavy chain. Release of clathrin in association with $\mathrm{Hsc} 70 * \mathrm{ADP}$ follows, and the subsequent, persistent association of clathrin with $\mathrm{Hsc} 70$ is important to prevent aberrant clathrin polymerization. Thus, the two canonical functions of Hsp70-dissociation of existing protein complexes or aggregates, and binding to a protein to inhibit its inappropriate aggregation-are recapitulated in uncoating. Association of clathrin with Hsc70 in vivo is regulated by Hsp110, an Hsp70 NEF that is itself a member of the Hsp70 family. How Hsp110 activity is itself regulated to make Hsc70-free clathrin available for endocytosis is unclear, though at synapses it's possible that the influx of calcium that accompanies depolarization activates the $\mathrm{Ca}^{++} /$calmodulin dependent calcineurin phosphatase which then dephosphorylates and activates Hsp110 to stimulate ADP/ATP exchange and release clathrin from Hsc70*ADP:clathrin complexes.

Keywords: clathrin, endocytosis, chaperone, NEF, Hsp70, Hsc70, Hsp110, auxilin

\section{The Uncoating ATPase}

In 1984 the Rothman lab reported identification of an "uncoating ATPase" (Braell et al., 1984; Schlossman et al., 1984), an abundant $70 \mathrm{kD}$ protein required to uncoat the clathrin coated vesicles that are the transient products of clathrin-mediated endocytosis at plasma membranes and clathrinmediated intracellular traffic involving Golgi, endosomes, and lysosomes (Figure 1). The ATPase activity of the $70 \mathrm{kD}$ protein was reported to be stimulated by clathrin and, by stabilizing coats with low $\mathrm{pH}$ or high $\mathrm{Mg}^{++}$concentrations so that uncoating was blocked, it could be shown that clathrin binding by the uncoating enzyme (and stimulation of its ATPase activity) preceded the uncoating step. They showed further that the uncoating enzyme dissociated the coats into clathrin triskelia and remained associated with the triskelia after uncoating, thereby sequestering and blocking the

Abbreviations: Hsc70, constitutive heat-shock protein 70; Hsp70, heat-shock protein 70; NEF, nucleotide exchange factor;

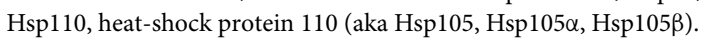




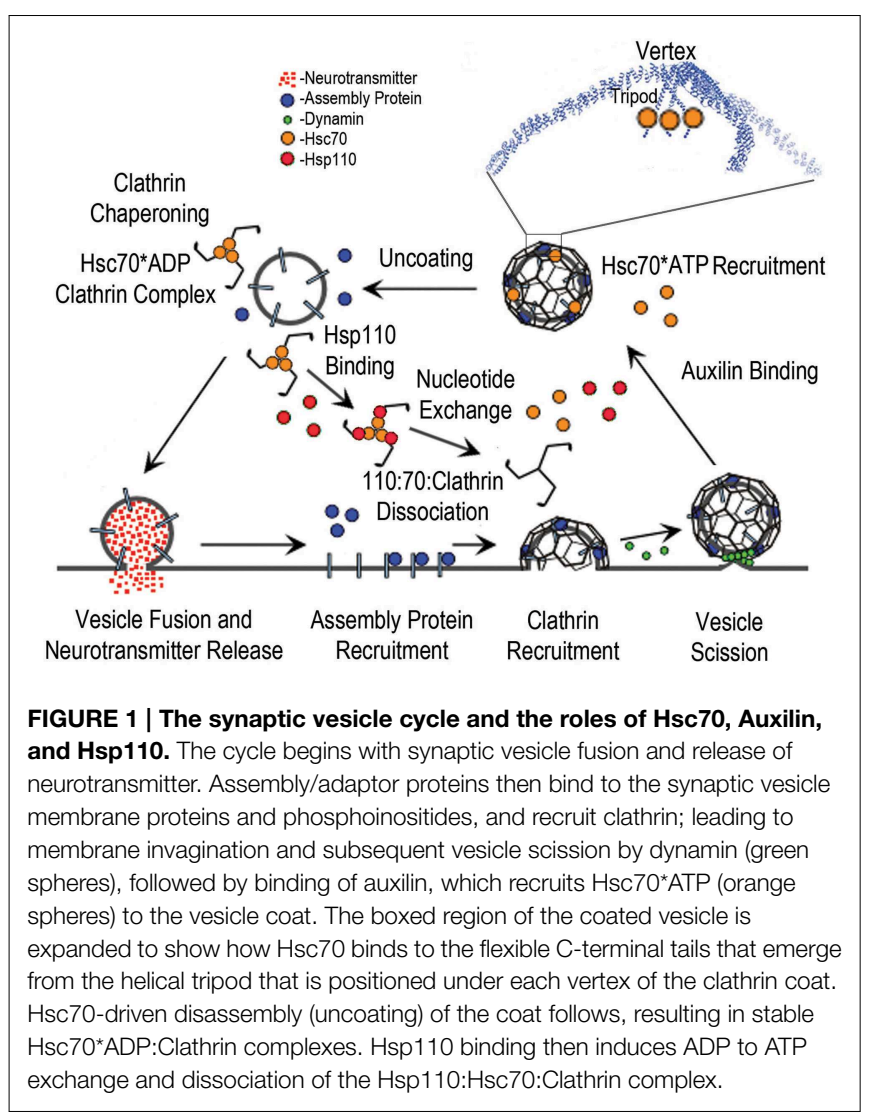

reassembly of the triskelia into coats. Shortly thereafter, Ungewickell reported in 1985 that the uncoating ATPase appeared to be a member of the Hsp70 stress-protein family (Ungewickell, 1985).

Subsequent work by the Ungewickell group led to identification of another protein, dubbed auxilin, that associated with and promoted the assembly of clathrin coats (Ahle and Ungewickell, 1990). Auxilin was shown to be identical to a protein identified by the Eisenberg lab to be required for the Hsc70 mediated disassembly of clathrin coats (Prasad et al., 1993). Auxilin was shown to be a J cochaperone which-like DnaJ, the founding member of the $J$ cochaperone familycontains a J domain which binds Hsc70*ATP (Schroder et al., 1995; Ungewickell et al., 1995; Holstein et al., 1996). The presence of both J- and clathrin-binding domains in auxilin provides the mechanism by which $\mathrm{Hsc70}$ is recruited to the clathrin coat to subsequently drive its disassembly, with auxilin acting catalytically as each auxilin molecule is able to recruit multiple Hsc70 molecules to the coated vesicle (Barouch et al., 1997). The Eisenberg group showed further that uncoating followed burst kinetics: addition of limiting amounts of Hsc70 to clathrin coats resulted in an initial, rapid burst of uncoating, followed by a slow steady-state rate (Barouch et al., 1994). These kinetics were attributed to Hsc70*ADP becoming stably associated with the released triskelia, so that it only becomes available to carry out further rounds of uncoating upon the slow release of ADP. ADP release then allows ATP to bind and stimulate release of Hsc70 from the clathrin.
Auxilin homologs were subsequently identified in several species including yeast, C. elegans, and mammals (Gall et al., 2000; Umeda et al., 2000; Greener et al., 2001). Mammalian forms include the neuronal-specific auxilin 1 and the ubiquitously expressed auxilin 2 (also called cyclin $\underline{G}$-associated protein kinase, or GAK) (Ahle and Ungewickell, 1990; Ungewickell et al., 1995; Kanaoka et al., 1997; Kimura et al., 1997; Greener et al., 2000; Umeda et al., 2000). While the amino acid sequences of auxilins are somewhat divergent between species, they all contain both clathrin binding and J domains (Gall et al., 2000; Greener et al., 2000, 2001; Pishvaee et al., 2000; Lemmon, 2001). Interestingly however, structural studies of auxilin led to the discovery that the structural diversity amongst $J$ domains is greater than was initially anticipated: the $\sim 100$ amino acid auxilin J domain is, by comparison to the canonical $\sim 70$ residue domain exemplified by DnaJ, extended by insertion of a long but structured loop which contributes to the interface with Hsc70 (Jiang et al., 2003). The roles of J-domain structural diversity in the specificity and regulation of Hsp70 function are still poorly understood (Hennessy et al., 2005).

A series of genetic and acute perturbation studies subsequently extended these biochemical studies in vivo (Pishvaee et al., 2000; Greener et al., 2001; Morgan et al., 2001; Newmyer and Schmid, 2001; Lee et al., 2005, 2006; Massol et al., 2006; Yim et al., 2010) and demonstrated that, despite the initial surprise at attributing the specialized function of vesicle uncoating to the generalist Hsc70 chaperone, vesicle uncoating both in vitro and in vivo follows what we would now describe as a canonical Hsp70 mechanism (Sousa, 2014) in which a J cochaperone binds Hsp70*ATP and delivers it to a substrate protein concomitant with ATP hydrolysis, formation of a stable Hsp $70 *$ ADP:protein substrate complex and release of the $J$ cochaperone:Hsp70 interaction. More recent studies have shown that recapitulation of this canonical mechanism extends even to the structural details of the clathrin:Hsc70 interaction. The Hsc70 binding site on the clathrin heavy chain has been mapped to a typical Hsc70 binding sequence ("QLMLT") which is present in an extended, flexible tail that emerges from the C-termini of each of the three helices that associate in each triskelion to form a helical tripod on the inner surface of each vertex of the clathrin coat (Figure 1) (Fotin et al., 2004; Rapoport et al., 2008; Xing et al., 2010).

\section{Models for Hsc70 Mediated Clathrin Coat Disassembly}

While the process of auxilin-mediated Hsc70 recruitment to the clathrin coat is now well understood, the mechanism by which Hsc70, once delivered to the coat, drives its disassembly is less clear and somewhat controversial. The Smith group has proposed a sequential disassembly mechanism in which auxilin first recruits an Hsc70 molecule to each of the three clathrin heavy chains that comprise each triskelion in a coat structure (Rothnie et al., 2011). Only after 3 Hsc70s have been loaded is the triskelion released from the coat assembly. In contrast, the Kirchhausen group has obtained evidence for a mechanism in which coat disassembly can be initiated with a substoichiometric 
number of Hsc70s, with as few as 1 Hsc70s for every $\sim 6$ clathrin heavy chains being sufficient to initiate uncoating (Bocking et al., 2011). Moreover, this number itself is variable: clathrin coats become more stable at lower $\mathrm{pH}$ and it was seen that, under such conditions, more Hsc70s had to bind to initiate disassembly. Conversely, coats destabilized by higher $\mathrm{pH}$ or mutations that disrupted clathrin:clathrin interactions required fewer Hsc70s to bind before disassembly was observed (Bocking et al., 2014). The mechanism proposed by the Kirchhausen group also differed from that of the Smith group in being non-sequential: coat disassembly was observed to begin when a certain number of Hsc70s (determined by $\mathrm{pH}$ and ionic conditions which modulate coat stability) had bound the coat, but Hsc70s continued to be recruited to the coat and further accelerated its disassembly even after disassembly had begun (Bocking et al., 2011). Though the Kirchhausen group developed their mechanism based on single-molecule experiments, while the Smith lab used data from ensemble experiments which can obscure mechanistic details, the latter group's conclusion are more consistent with early data (Schmid and Rothman, 1985) indicating a 1:1 stoichiometric association of Hsc70 with clathrin heavy chain during uncoating. However, it appears likely that the Kirchhausen group is correct in their conclusions that coats can be disassembled with fewer than stoichiometric numbers of Hsc70s bound. The observation of stoichiometric binding may well be a consequence of the fact that, at high Hsc70 concentrations, auxilin recruits the chaperone to the coats faster than the coats themselves disassemble (Bocking et al., 2011). As a consequence, at high Hsc70 concentrations the end state of the disassembly reaction is triskelia with Hsc70s bound 1:1 to clathrin heavy chain (3 Hsc70s per triskelion), but experiments carried out at lower Hsc70 concentrations reveal that coats can be disassembled with substoichiometric numbers of $\mathrm{Hsc} 70$.

Based on their structural studies of Hsc70s bound to clathrin coats, the Harrison and Kirchhausen groups proposed further that the bound Hsc70s drive disassembly by a "Brownian/Steric Wedge" mechanism. They suggested that, even in the absence of Hsc70, coats are always experiencing spontaneous fluctuations that loosen interactions between triskelia, but that such fluctuations never accumulate to a point that leads to coat disassembly (Xing et al., 2010). However, when Hsc70s are bound to the C-terminal tails under each coat vertex, they sterically block reversal of these loosening fluctuations, which then accumulate to a point where they result in coat disassembly. A different model, based on the excluded volume/entropic pulling mechanism proposed by De Los Rios and Goloubinoff (De Los Rios et al., 2006; Goloubinoff and De Los Rios, 2007) to explain how Hsp70s move proteins through channels or dissociate protein aggregates, has also been advanced. This model suggests that it is not the ability of Hsc70s to act as a passive, steric wedge that causes disassembly, but the fact that they are bound under each coat vertex by association with flexible polypeptide tethers. Such flexible tethering allows the Hsc70s to generate a disassembling force through intermolecular collisions with the walls of the coat (Lafer et al., 2014). In contrast to the "steric wedge" model, this might be described as a "wrecking ball" model for coat disassembly. Determination of which of these mechanistic models is correct awaits further experimentation.

\section{Control of Hsc70:Clathrin Chaperoning by Hsp110}

The highly specialized uncoating reaction may provide insight into the mechanism of the more general proteostatic functions of the Hsp70 chaperones. Indeed, Hsc70 plays at least two roles in uncoating that may be considered analogous to its functions in supporting native protein folding. First, the disassembly of the clathrin coat may be considered analogous to reactions in which Hsp70s dissociate aggregates of misfolded proteins (Goloubinoff et al., 1999; Diamant et al., 2000; Ben-Zvi and Goloubinoff, 2001; Ben-Zvi et al., 2004; Shorter, 2011; Rampelt et al., 2012). Second, once disassembled, Hsc70 remains associated with triskelia (Schuermann et al., 2008) and inhibits their aberrant polymerization; i.e., Hsc70 not only disassembles coats, it also chaperones triskelia (Figure 1) via a mechanism that may be considered analogous to that by which Hsp70s sequester and inhibit aggregation of misfolded proteins (Mogk et al., 1999). Indeed, just as mutations in Hsp70 can accelerate accumulation of protein aggregates in vivo (Hesterkamp and Bukau, 1998), mutations in Hsc70 lead to aberrant clathrin polymerization and defects in endocytosis (Newmyer and Schmid, 2001).

The conclusion that Hsc70 not only disassembles clathrin coats, but also sequesters depolymerized clathrin implies that this chaperoning activity must be regulated so that clathrin can be released and made available as required for endocytosis. The most likely candidates for such regulators are the Hsp70 nucleotide exchange factors (NEFs), which control the association of Hsp70s with their protein substrates by stimulating the release of ADP from the Hsp70 (Packschies et al., 1997). This allows ATP to bind, which stimulates release of the substrate from the otherwise very stable Hsp70^ADP:protein substrate complex (Mccarty et al., 1995; Takeda and Mckay, 1996; Theyssen et al., 1996). In vitro, NEFs have been shown to release Hsc70 from triskelia and to accelerate the slow steady-state rate of uncoating that follows addition of limiting amounts of Hsc70 to reactions with clathrin coats and ATP (Schuermann et al., 2008), consistent with the Eisenberg group's conclusion that these burst kinetics reflect formation of stable $\mathrm{Hsc} 70^{\star} \mathrm{ADP}$ :clathrin complexes (Barouch et al., 1994). In vitro, both the Bagl and Hsp110 NEFs could accelerate steady-state uncoating and Hsc70:clathrin dissociation, leaving it unclear which of these NEFs regulates clathrin:Hsc70 association in vivo. Recent experiments indicate that the relevant in vivo NEF is likely to be Hsp110, a protein that is itself a member of the Hsp70 family and the most abundant Hsp70 NEF in vertebrate brain (Morgan et al., 2013). Acute inhibition of Hsp110 at lamprey giant reticulospinal synapses was shown to inhibit endocytosis (specifically, synaptic vesicle recycling), presumably because inhibition of Hsp110 blocked its ability to stimulate nucleotide exchange and thereby blocked release of clathrin from Hsc70 (Morgan et al., 2013).

The question of how Hsc70:clathrin association is controlled so as to coordinate clathrin availability with endocytic activity may therefore reduce, at least in part, to the question of how Hsp110 activity is regulated. Currently, answers to this question must remain largely speculative, but one possible mechanism involves the dephosphin hypothesis (Cousin and Robinson, 2001) that has been developed to explain how the depolarization 
induced calcium influx that occurs at nerve terminals during synaptic transmission stimulates endocytosis. This hypothesis proposes that a coordinated wave of dephosphorylation of a number of proteins required for endocytosis (the "dephosphins" dynamin I, amphiphysins I and II, synaptojanin, epsin, eps15 and AP180) (Cousin et al., 2001) is driven by $\mathrm{Ca}^{++}$activation of the calcium/calmodulin dependent protein phophatase calcineurin. Dephosphorylation of these proteins is proposed to stimulate their activity and thereby drive endocytosis.

Could such a mechanism regulate availability of Hsc70-free clathrin for endocytosis? Hsp110 is known to be phosphorylated, primarily at serine residues, both in vivo and in vitro (Ishihara et al., 2000). Evidence suggests that the relevant in vivo kinase(s) may be casein kinase II (CKII) or a kinase that has CKII-like specificity. In vitro, CKII phosphorylates mouse Hsp110 at S509, and this residue is also phosphorylated in vivo (Ishihara et al., 2003). Phosphorylation at S509 relieves the inhibitory effect of Hsp110 on Hsp70-mediated luciferase refolding in vitro, and an Hsp110 S509A mutant suppresses Hsp70-mediated luciferase refolding in vivo.

These observations require further comment as Hsp110 is usually observed, and is expected to, stimulate, rather than inhibit, Hsp70 functions. However, the net effect of stimulation of Hsp70 nucleotide exchange by Hsp110 is to shorten the lifetime of the Hsp70:protein substrate complex. If we consider an Hsp70-mediated reaction, such as refolding of misfolded proteins, it is easy to imagine that there will be an optimal lifetime for this complex that balances the reduction in second order processes like aggregation due to sequestration of misfolded proteins with Hsp70, and the rate of first order processes like refolding, which require that the misfolded protein be released from the Hsp70. Indeed, it is observed that when Hsp110 is titrated into Hsp70-mediated protein refolding reactions, there is an Hsp110 concentration that drives maximal refolding, and that Hsp110 concentrations in excess of this can slow refolding (Dragovic et al., 2006; Tzankov et al., 2008; Rampelt et al., 2012).

Since, in the experiments described above, phosphorylation of Hsp110 was observed to relieve inhibition of Hsp70-mediated luciferase refolding, this indicates that these experiments were carried out under conditions where Hsp110 was inducing Hsp70 nucleotide exchange and protein substrate release to a degree that inhibited protein refolding. This implies that phosphorylation of Hsp110 reduces its ability to act as an Hsp70 NEF, and suggests the following mechanism for how $\mathrm{Ca}^{++} /$calmodulin activation of the calcineurin phosphatase causes Hsc70 to release clathrin and make it available for endocytosis: in a resting synapse, clathrin may be mostly sequestered by Hsc70, which is in excess of clathrin (Morgan et al., 2013). Hsp110 will be phosphorylated (the brain has been shown to be exceptionally rich in the phosphorylated form of Hsp110 Ishihara et al., 2000), and its NEF activity will be correspondingly repressed. Upon depolarization, the influx of $\mathrm{Ca}^{++}$into the pre-synaptic terminal will activate calmodulin and calcineurin, leading to a wave of dephosphorylation of multiple proteins, including Hsp110. Dephosphorylation of Hsp110 will stimulate its NEF activity and ultimately lead to increased release of clathrin from Hsc70, thus

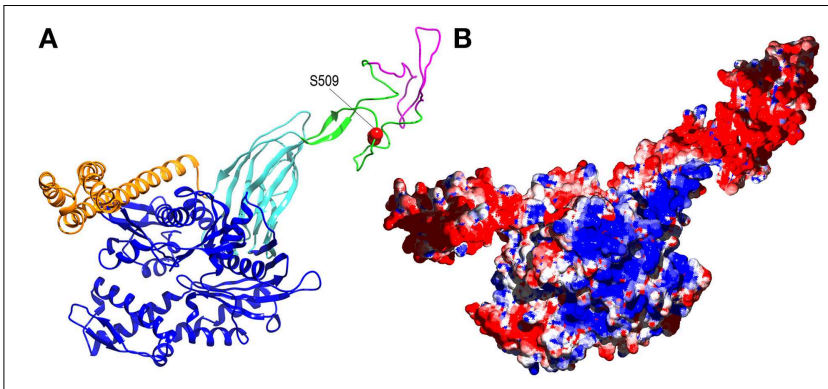

FIGURE 2 | Alternative splicing and phosphorylation of the acidic insertion loop may regulate Hsp110 activity. (A) Ribbon model of human Hsp110 based on the crystal structure of yeast Hsp110 (pbd 3C7N Schuermann et al., 2008) with nucleotide binding domain in blue, the $\beta$-sandwich and $\alpha$-helical portions of the protein binding domain in, respectively, cyan and orange, and the acidic insertion loop in green with the element that is removed by alternative splicing in magenta and the phosphorylated S509 side-chain in red. (B) The same model as in A but in surface representation with positively and negatively charged regions in blue and red, respectively.

driving the clathrin-mediated endocytic retrieval of the synaptic vesicle proteins that follows the fusion of the synaptic vesicle with the plasma membrane and release of neurotransmitter (Figure 1).

How might phosphorylation regulate Hsp110 NEF activity? Again, any answer must be speculative, but it is striking that the phosphorylated S509 residue sits within the flexible $\sim 95$ residue acidic insertion loop that is positioned near the end of the $\beta$-sandwich segment of the Hsp110 protein binding domain (Figure 2A). The acidic insertion loop uniquely distinguishes the Hsp110 proteins from the more distantly related members of the Hsp70 family. There is some evidence that this loop may regulate Hsp110: the Hendershot lab observed that deletions in the insertion loop of ER Hsp110 (Grp170) affect its ability to interact with protein substrates (Pereira et al., 2014), and it has been found that the RNA encoding this loop undergoes alternative splicing (Yasuda et al., 1995). The full-length version of mouse Hsp110 (also called Hsp105 $\alpha$ as Hsp110 has also been designated Hsp105) is apparently the predominant, constitutively expressed version of the protein, while the alternatively spliced Hsp $105 \beta$, which may be expressed upon heat shock (Hatayama et al., 1994), is missing 44 residues from the loop (Figure 2A). There is no information on how this loop might regulate Hsp110 activity, but the loop's flexibility and negative charge could allow it to interact with multiple electropositive regions on the Hsp110 (or Hsp70) surface (Figure 2B), and phosphorylation might modulate these ionic interactions. All of these highly speculative mechanisms await testing by future experimentation.

\section{Summary}

Hsc70 disassembly of clathrin coats follows a canonical Hsp70 mechanism in which a J cochaperone binds Hsp70*ATP and transfers it to a substrate, concomitant with ATP hydrolysis to form a stable Hsp70*ADP:substrate complex. After disassembling the coat, $\mathrm{Hsc70}$ remains associated with 
clathrin to prevent its aberrant polymerization. When required for endocytosis clathrin is released from Hsc70 by a nucleotide exchange factor, whose activity may be regulated by $\mathrm{Ca}^{++}$ dependent dephosphorylation.

\section{References}

Ahle, S., and Ungewickell, E. (1990). Auxilin, a newly identified clathrin-associated protein in coated vesicles from bovine brain. J. Cell Biol. 111, 19-29. doi: 10.1083/jcb.111.1.19

Barouch, W., Prasad, K., Greene, L. E., and Eisenberg, E. (1994). ATPase activity associated with the uncoating of clathrin baskets by Hsp70. J. Biol. Chem. 269, 28563-28568.

Barouch, W., Prasad, K., Greene, L., and Eisenberg, E. (1997). Auxilininduced interaction of the molecular chaperone $\mathrm{Hsc} 70$ with clathrin baskets. Biochemistry 36, 4303-4308. doi: 10.1021/bi962727z

Ben-Zvi, A., De Los Rios, P., Dietler, G., and Goloubinoff, P. (2004). Active solubilization and refolding of stable protein aggregates by cooperative unfolding action of individual hsp70 chaperones. J. Biol. Chem. 279, 37298-37303. doi: 10.1074/jbc.M405627200

Ben-Zvi, A. P., and Goloubinoff, P. (2001). Review: mechanisms of disaggregation and refolding of stable protein aggregates by molecular chaperones. J. Struct. Biol. 135, 84-93. doi: 10.1006/jsbi.2001.4352

Bocking, T., Aguet, F., Harrison, S. C., and Kirchhausen, T. (2011). Single-molecule analysis of a molecular disassemblase reveals the mechanism of Hsc70driven clathrin uncoating. Nat. Struct. Mol. Biol. 18, 295-301. doi: 10.1038/ns mb. 1985

Bocking, T., Aguet, F., Rapoport, I., Banzhaf, M., Yu, A., Zeeh, J. C., et al. (2014). Key interactions for clathrin coat stability. Structure 22, 819-829. doi: 10.1016/j.str.2014.04.002

Braell, W. A., Schlossman, D. M., Schmid, S. L., and Rothman, J. E. (1984). Dissociation of clathrin coats coupled to the hydrolysis of ATP: role of an uncoating ATPase. J. Cell Biol. 99, 734-741. doi: 10.1083/jcb.99.2.734

Cousin, M. A., and Robinson, P. J. (2001). The dephosphins: dephosphorylation by calcineurin triggers synaptic vesicle endocytosis. Trends Neurosci. 24, 659-665. doi: 10.1016/S0166-2236(00)01930-5

Cousin, M. A., Tan, T. C., and Robinson, P. J. (2001). Protein phosphorylation is required for endocytosis in nerve terminals: potential role for the dephosphins dynamin I and synaptojanin, but not AP180 or amphiphysin. J. Neurochem. 76, 105-116. doi: 10.1046/j.1471-4159.2001.00049.x

De Los Rios, P., Ben-Zvi, A., Slutsky, O., Azem, A., and Goloubinoff, P. (2006). Hsp70 chaperones accelerate protein translocation and the unfolding of stable protein aggregates by entropic pulling. Proc. Natl. Acad. Sci. U.S.A. 103, 6166-6171. doi: 10.1073/pnas.0510496103

Diamant, S., Ben-Zvi, A. P., Bukau, B., and Goloubinoff, P. (2000). Size-dependent disaggregation of stable protein aggregates by the DnaK chaperone machinery. J. Biol. Chem. 275, 21107-21113. doi: 10.1074/jbc.M001293200

Dragovic, Z., Broadley, S. A., Shomura, Y., Bracher, A., and Hartl, F. U. (2006). Molecular chaperones of the Hsp110 family act as nucleotide exchange factors of Hsp70s. EMBO J. 25, 2519-2528. doi: 10.1038/sj.emboj.7601138

Fotin, A., Cheng, Y., Sliz, P., Grigorieff, N., Harrison, S. C., Kirchhausen, T., et al. (2004). Molecular model for a complete clathrin lattice from electron cryomicroscopy. Nature 432, 573-579. doi: 10.1038/nature03079

Gall, W. E., Higginbotham, M. A., Chen, C., Ingram, M. F., Cyr, D. M., and Graham, T. R. (2000). The auxilin-like phosphoprotein Swa2p is required for clathrin function in yeast. Curr. Biol. 10, 1349-1358. doi: 10.1016/S09609822(00)00771-5

Goloubinoff, P., and De Los Rios, P. (2007). The mechanism of Hsp70 chaperones: (entropic) pulling the models together. Trends Biochem. Sci. 32, 372-380. doi: 10.1016/j.tibs.2007.06.008

Goloubinoff, P., Mogk, A., Zvi, A. P., Tomoyasu, T., and Bukau, B. (1999). Sequential mechanism of solubilization and refolding of stable protein aggregates by a bichaperone network. Proc. Natl. Acad. Sci. U.S.A. 96, 13732-13737. doi: 10.1073/pnas.96.24.13732

\section{Acknowledgments}

Work in the EL and RS laboratories is supported by NIH-NINDS grant NS029051.

Greener, T., Grant, B., Zhang, Y., Wu, X., Greene, L. E., Hirsh, D., et al. (2001) Caenorhabditis elegans auxilin: a J-domain protein essential for clathrinmediated endocytosis in vivo. Nat. Cell Biol. 3, 215-219. doi: 10.1038/350 55137

Greener, T., Zhao, X., Nojima, H., Eisenberg, E., and Greene, L. E. (2000). Role of cyclin G-associated kinase in uncoating clathrin-coated vesicles from non-neuronal cells. J. Biol. Chem. 275, 1365-1370. doi: 10.1074/jbc.275.2.1365

Hatayama, T., Yasuda, K., and Nishiyama, E. (1994). Characterization of highmolecular-mass heat shock proteins and 42 degrees C-specific heat shock proteins of murine cells. Biochem. Biophys. Res. Commun. 204, 357-365. doi: 10.1006/bbrc.1994.2467

Hennessy, F., Nicoll, W. S., Zimmermann, R., Cheetham, M. E., and Blatch, G. L. (2005). Not all J domains are created equal: implications for the specificity of Hsp40-Hsp70 interactions. Protein Sci. 14, 1697-1709. doi: $10.1110 /$ ps.051406805

Hesterkamp, T., and Bukau, B. (1998). Role of the DnaK and HscA homologs of Hsp70 chaperones in protein folding in E. coli. EMBO J. 17, 4818-4828. doi: 10.1093/emboj/17.16.4818

Holstein, S. E., Ungewickell, H., and Ungewickell, E. (1996). Mechanism of clathrin basket dissociation: separate functions of protein domains of the DnaJ homologue auxilin. J. Cell Biol. 135, 925-937. doi: 10.1083/jcb.135.4.925

Ishihara, K., Yamagishi, N., and Hatayama, T. (2003). Protein kinase CK2 phosphorylates Hsp105 alpha at Ser509 and modulates its function. Biochem. J. 371, 917-925. doi: 10.1042/BJ20021331

Ishihara, K., Yasuda, K., and Hatayama, T. (2000). Phosphorylation of the 105 $\mathrm{kDa}$ heat shock proteins, HSP105alpha and HSP105beta, by casein kinase II. Biochem. Biophys. Res. Commun. 270, 927-931. doi: 10.1006/bbrc.2000.2541

Jiang, J., Taylor, A. B., Prasad, K., Ishikawa-Brush, Y., Hart, P. J., Lafer, E. M., et al. (2003). Structure-function analysis of the auxilin J-domain reveals an extended Hsc70 interaction interface. Biochemistry 42, 5748-5753. doi: $10.1021 /$ bi034270g

Kanaoka, Y., Kimura, S. H., Okazaki, I., Ikeda, M., and Nojima, H. (1997). GAK a cyclin $\mathrm{G}$ associated kinase contains a tensin/auxilin-like domain. FEBS Lett. 402, 73-80. doi: 10.1016/S0014-5793(96)01484-6

Kimura, S. H., Tsuruga, H., Yabuta, N., Endo, Y., and Nojima, H. (1997). Structure, expression, and chromosomal localization of human GAK. Genomics 44, 179-187. doi: 10.1006/geno.1997.4873

Lafer, E. M., Jin, S., and Sousa, R. (2014). "Hsc70 disassembly of clathrin cages driven by osmotic force amplified by chaperone self-association," in Society for Neuroscience Annual Meeting 2014 (Washington, DC). Program No. 403.09.

Lee, D. W., Wu, X., Eisenberg, E., and Greene, L. E. (2006). Recruitment dynamics of GAK and auxilin to clathrin-coated pits during endocytosis. J. Cell Sci. 119, 3502-3512. doi: 10.1242/jcs.03092

Lee, D. W., Zhao, X., Zhang, F., Eisenberg, E., and Greene, L. E. (2005). Depletion of GAK/auxilin 2 inhibits receptor-mediated endocytosis and recruitment of both clathrin and clathrin adaptors. J. Cell Sci. 118, 4311-4321. doi: $10.1242 /$ jcs. 02548

Lemmon, S. K. (2001). Clathrin uncoating: auxilin comes to life. Curr. Biol. 11, R49-R52. doi: 10.1016/S0960-9822(01)00010-0

Massol, R. H., Boll, W., Griffin, A. M., and Kirchhausen, T. (2006). A burst of auxilin recruitment determines the onset of clathrin-coated vesicle uncoating. Proc. Natl. Acad. Sci. U.S.A. 103, 10265-10270. doi: 10.1073/pnas.0603369103

Mccarty, J. S., Buchberger, A., Reinstein, J., and Bukau, B. (1995). The role of ATP in the functional cycle of the DnaK chaperone system. J. Mol. Biol. 249, 126-137. doi: 10.1006/jmbi.1995.0284

Mogk, A., Tomoyasu, T., Goloubinoff, P., Rudiger, S., Roder, D., Langen, H., et al. (1999). Identification of thermolabile Escherichia coli proteins: prevention and reversion of aggregation by DnaK and ClpB. EMBO J. 18, 6934-6949. doi: 10.1093/emboj/18.24.6934 
Morgan, J. R., Jiang, J., Oliphint, P. A., Jin, S., Gimenez, L. E., Busch, D. J., et al. (2013). A role for an hsp70 nucleotide exchange factor in the regulation of synaptic vesicle endocytosis. J. Neurosci. 33, 8009-8021. doi: 10.1523/JNEUROSCI.4505-12.2013

Morgan, J. R., Prasad, K., Jin, S., Augustine, G. J., and Lafer, E. M. (2001). Uncoating of clathrin-coated vesicles in presynaptic terminals: roles for Hsc70 and auxilin. Neuron 32, 289-300. doi: 10.1016/S0896-6273(01)00467-6

Newmyer, S. L., and Schmid, S. L. (2001). Dominant-interfering Hsc70 mutants disrupt multiple stages of the clathrin-coated vesicle cycle in vivo. J. Cell Biol. 152, 607-620. doi: 10.1083/jcb.152.3.607

Packschies, L., Theyssen, H., Buchberger, A., Bukau, B., Goody, R. S., and Reinstein, J. (1997). GrpE accelerates nucleotide exchange of the molecular chaperone DnaK with an associative displacement mechanism. Biochemistry 36, 3417-3422. doi: 10.1021/bi9628351

Pereira, E. R., Frudd, K., Awad, W., and Hendershot, L. M. (2014). Endoplasmic reticulum (ER) stress and hypoxia response pathways interact to potentiate hypoxia-inducible factor 1 (HIF-1) transcriptional activity on targets like vascular endothelial growth factor (VEGF). J. Biol. Chem. 289, 3352-3364. doi: 10.1074/jbc.M113.507194

Pishvaee, B., Costaguta, G., Yeung, B. G., Ryazantsev, S., Greener, T., Greene, L. E., et al. (2000). A yeast DNA J protein required for uncoating of clathrin-coated vesicles in vivo. Nat. Cell Biol. 2, 958-963. doi: 10.1038/35046619

Prasad, K., Barouch, W., Greene, L., and Eisenberg, E. (1993). A protein cofactor is required for uncoating of clathrin baskets by uncoating ATPase. J. Biol. Chem. 268, 23758-23761.

Rampelt, H., Kirstein-Miles, J., Nillegoda, N. B., Chi, K., Scholz, S. R., Morimoto, R. I., et al. (2012). Metazoan Hsp70 machines use Hsp110 to power protein disaggregation. EMBO J. 31, 4221-4235. doi: 10.1038/emboj.2012.264

Rapoport, I., Boll, W., Yu, A., Bocking, T., and Kirchhausen, T. (2008). A motif in the clathrin heavy chain required for the hsc70/auxilin uncoating reaction. Mol. Biol. Cell 19, 405-413. doi: 10.1091/mbc.E07-09-0870

Rothnie, A., Clarke, A. R., Kuzmic, P., Cameron, A., and Smith, C. J. (2011). A sequential mechanism for clathrin cage disassembly by $70-\mathrm{kDa}$ heatshock cognate protein (Hsc70) and auxilin. Proc. Natl. Acad. Sci. U.S.A. 108, 6927-6932. doi: 10.1073/pnas. 1018845108

Schlossman, D. M., Schmid, S. L., Braell, W. A., and Rothman, J. E. (1984). An enzyme that removes clathrin coats: purification of an uncoating ATPase. J. Cell Biol. 99, 723-733. doi: 10.1083/jcb.99.2.723

Schmid, S. L., and Rothman, J. E. (1985). Enzymatic dissociation of clathrin cages in a two-stage process. J. Biol. Chem. 260, 10044-10049.

Schroder, S., Morris, S. A., Knorr, R., Plessmann, U., Weber, K., Nguyen, G. V., et al. (1995). Primary structure of the neuronal clathrin-associated protein auxilin and its expression in bacteria. Eur. J. Biochem. 228, 297-304. doi: 10.1111/j.1432-1033.1995.tb20263.x

Schuermann, J. P., Jiang, J., Cuellar, J., Llorca, O., Wang, L., Gimenez, L. E., et al. (2008). Structure of the Hsp110:Hsc70 nucleotide exchange machine. Mol. Cell 31, 232-243. doi: 10.1016/j.molcel.2008.05.006
Shorter, J. (2011). The mammalian disaggregase machinery: Hsp110 synergizes with Hsp70 and Hsp40 to catalyze protein disaggregation and reactivation in a cell-free system. PLOS ONE 6:e26319. doi: 10.1371/journal.pone. 0026319

Sousa, R. J. (2014). Structural mechanisms of chaperone mediated protein disaggregation. Front. Mol. Biosci. 1:12. doi: 10.3389/fmolb.2014. 00012

Takeda, S., and Mckay, D. B. (1996). Kinetics of peptide binding to the bovine $70 \mathrm{kDa}$ heat shock cognate protein, a molecular chaperone. Biochemistry 35, 4636-4644. doi: 10.1021/bi952903o

Theyssen, H., Schuster, H. P., Packschies, L., Bukau, B., and Reinstein, J. (1996). The second step of ATP binding to DnaK induces peptide release. J. Mol. Biol. 263, 657-670. doi: 10.1006/jmbi.1996.0606

Tzankov, S., Wong, M. J., Shi, K., Nassif, C., and Young, J. C. (2008). Functional divergence between co-chaperones of Hsc70. J. Biol. Chem. 283, 27100-27109. doi: 10.1074/jbc.M803923200

Umeda, A., Meyerholz, A., and Ungewickell, E. (2000). Identification of the universal cofactor (auxilin 2) in clathrin coat dissociation. Eur. J. Cell Biol. 79, 336-342. doi: 10.1078/S0171-9335(04)70037-0

Ungewickell, E. (1985). The 70-kd mammalian heat shock proteins are structurally and functionally related to the uncoating protein that releases clathrin triskelia from coated vesicles. $E M B O$ J. 4, 3385-3391.

Ungewickell, E., Ungewickell, H., Holstein, S. E., Lindner, R., Prasad, K., Barouch, W., et al. (1995). Role of auxilin in uncoating clathrin-coated vesicles. Nature 378, 632-635. doi: 10.1038/378632a0

Xing, Y., Bocking, T., Wolf, M., Grigorieff, N., Kirchhausen, T., and Harrison, S. C. (2010). Structure of clathrin coat with bound Hsc70 and auxilin: mechanism of Hsc70-facilitated disassembly. EMBO J. 29, 655-665. doi: 10.1038/emboj.2009.383

Yasuda, K., Nakai, A., Hatayama, T., and Nagata, K. (1995). Cloning and expression of murine high molecular mass heat shock proteins, HSP105. J. Biol. Chem. 270, 29718-29723. doi: 10.1074/jbc.270.50.29718

Yim, Y. I., Sun, T., Wu, L. G., Raimondi, A., De Camilli, P., Eisenberg, E., et al. (2010). Endocytosis and clathrin-uncoating defects at synapses of auxilin knockout mice. Proc. Natl. Acad. Sci. U.S.A. 107, 4412-4417. doi: $10.1073 /$ pnas. 1000738107

Conflict of Interest Statement: The authors declare that the research was conducted in the absence of any commercial or financial relationships that could be construed as a potential conflict of interest.

Copyright (C) 2015 Sousa and Lafer. This is an open-access article distributed under the terms of the Creative Commons Attribution License (CC BY). The use, distribution or reproduction in other forums is permitted, provided the original author(s) or licensor are credited and that the original publication in this journal is cited, in accordance with accepted academic practice. No use, distribution or reproduction is permitted which does not comply with these terms. 